\title{
In vitro detection of adrenocorticotropic hormone levels by fluorescence correlation spectroscopy immunoassay for mathematical modeling of glucocorticoid-mediated feedback mechanisms
}

\author{
Martin Gerald Puchinger ${ }^{1 *}$, Clemens Alexander Zarzer ${ }^{2}$, Philipp Kügler ${ }^{2}$, Erwin Gaubitzer ${ }^{1}$ and Gottfried Köhler ${ }^{1}$
}

\begin{abstract}
Performing quantitative, highly sensitive measurements at a single molecule level is often necessary to address specific issues related to complex molecular and biochemical systems. For that purpose, we present a technique exploiting both the flexibility of immunoassays as well as the low operating costs and high throughput rates of the fluorescence correlation spectroscopy (FCS) method. That way we have established a quantitative measurement technique providing accurate and flexibly time resolved data of single molecules. Nanomolar changes in adrenocorticotropic hormone (ACTH) levels have been detected in a short time-frame that are caused by fast feedback actions in AtT-20 anterior pituitary glands in vitro. Especially with respect to clinical diagnostic or mathematical modeling this improved FCS setup may be of high relevance in order to accurately quantify the amounts of peptide hormones_-such as ACTH—as well as signaling molecules, transcription factors, etc., being involved in intra- and extracellular reaction networks.
\end{abstract}

Keywords: ACTH, FCS, AtT-20, Cortisol, CRH, Glucocorticoid membrane receptor, ODE model, Parameter identification

\section{Introduction}

Adrenocorticotropic hormone $(\mathrm{ACTH})$ is a 39-amino acid long straight-chain peptide hormone $(4.5 \mathrm{kDa})$ that is derived from a 266 -amino acid precursor pro-opiomelanocortin. It is secreted by the anterior pituitary gland and is considered one of the major stress hormones within the hypothalamic-pituitary-adrenal (HPA)-axis system: The hypothalamus secrets corticotrophinreleasing hormone $(\mathrm{CRH})$, which stimulates the release of ACTH in the corticotrophic anterior pituitary gland [1]. Consequently, ACTH causes the production of cortisol in the adrenal glands. However, beside corticotrophic feedback actions several other feedback controls on the metabolomic or genomic level provide a complex and multifaceted system. One of the most prominent and

\footnotetext{
* Correspondence: martin.puchinger@univie.ac.at

'Department of Structural and Computational Biology, Max F. Perutz Laboratories (MFPL), University of Vienna, Campus-Vienna-Biocenter 5, Vienna 1030, Austria

Full list of author information is available at the end of the article
}

well-studied feedback controls is the down-regulation of ACTH production by cortisol. The down-regulation is mediated via two feedback mechanisms working on a genomic and non-genomic levels (see Figure 1). Hence, we observe fast (within seconds to minutes) and slow (after several hours) negative feedback actions in response to the exposure with cortisol [2]. These feedback mechanisms are still subject of research and particularly their interplay is not fully understood. Hence, as ACTH represents the main response in regard to this glucocorticoid feedback, an accurate detection of in vitro extracellular ACTH concentration is of high relevance.

The fluorescence correlation spectroscopy (FCS) has proven to be a powerful tool for studying supramolecular associations [3,4], DNA hybridization reactions [5], and detecting single molecule concentrations [6,7]. Due to its high sensitivity, short analysis time and small sample volume requirements FCS have become a valuable tool in molecular biology. 


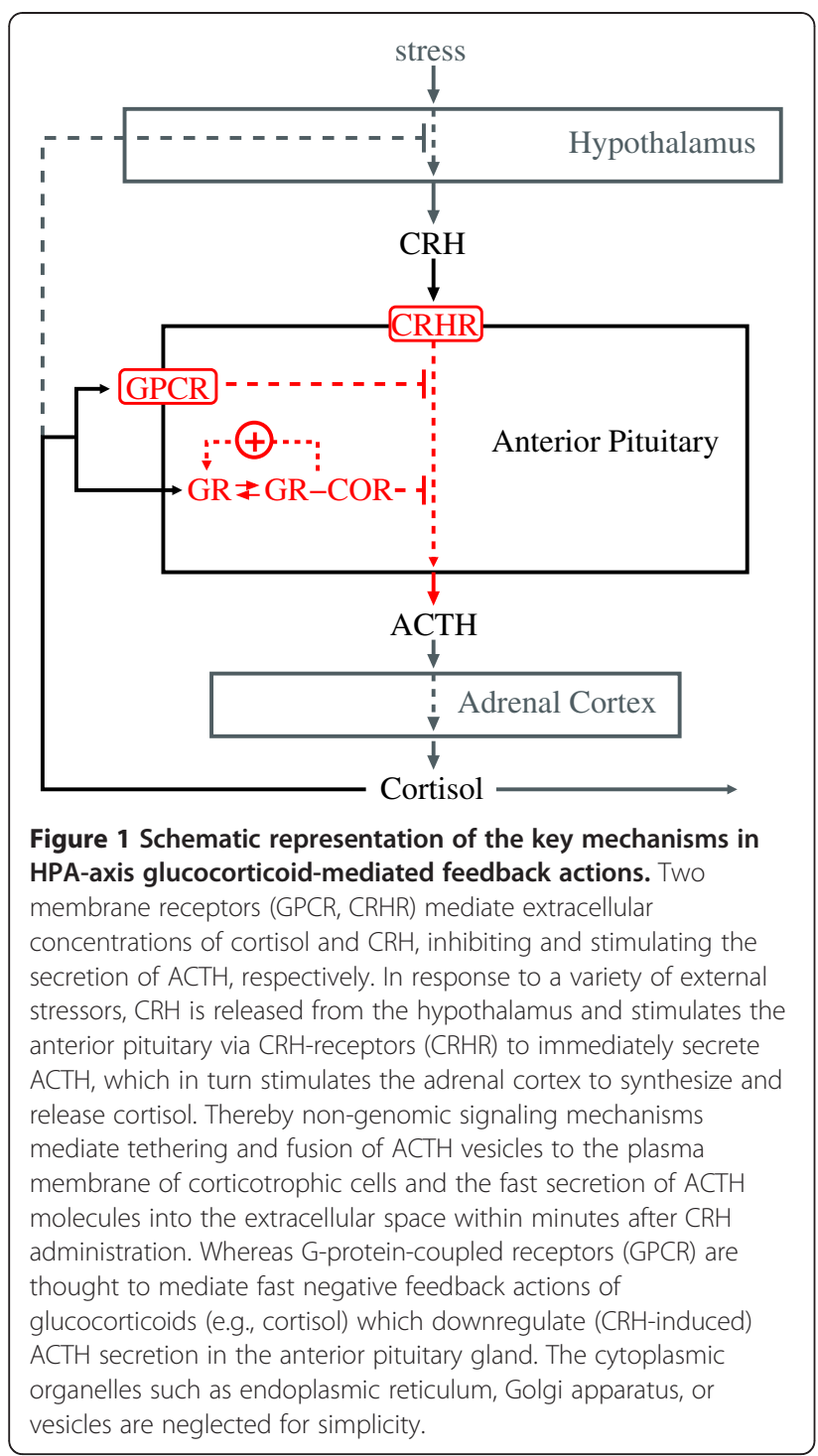

In this article, we present an improved FCS setup to detect nanomolar changes of peptides in vitro by combining the fast FCS technique [8] with the highly specific routines of an immunoassay. We exemplify this procedure by means of the in vitro measurement of the ACTH peptide secretion from AtT-20 mouse pituitary cells. Particularly, we use a labeled monoclonal antiACTH antibody (specific for the $N$-terminal epitope on the ACTH peptide) to capture the ACTH molecule, making it visible for the FCS. However, in order to detect low molecular weight peptides such as ACTH, the binding of a second unlabeled monoclonal antiACTH antibody to the C-terminal site of the ACTH peptide is necessary in order to cause a significant change in the diffusion time between the free labeled antibody and the $\operatorname{mAb}(N)$ $\mathrm{ACTH}-\mathrm{mAb}(\mathrm{C})$ immunocomplex. By measuring this discrepancy in the FCS, the concentration of the target peptide can accurately be determined.

\section{Materials and methods Cell culture}

The used AtT-20 cells (ATCC no. CCL-89) were purchased from the American Type Culture Collection (ATCC, Manassas, USA) and passaged at a subcultivation ratio of 1:4 every 5 days. Cells were seeded onto polystyrene 24-well tissue culture plates (Nalge Nunc International, Japan) at a density of $1.0 \times 10^{4}$ cells $/ \mathrm{ml}$, grown in Dulbecco's Modified Eagle's Medium (SigmaAldrich Inc., St. Louis, USA) supplemented with $10 \%$ fetal bovine serum, $1.5 \mathrm{~g} / \mathrm{l}$ sodium bicarbonate, 10 Units $/ \mathrm{ml}$ penicillin, and $10 \mu \mathrm{g} / \mathrm{ml}$ streptomycin, and maintained in an incubator (HERAcell ${ }^{\circledR}$, Thermo Scientific, USA) at $37^{\circ} \mathrm{C}, 6 \% \mathrm{CO}_{2}$ and $95 \%$ relative humidity. After 92 and $114 \mathrm{~h}$ of cell growing, AtT-20 cells were exposed to doses of $10 \mathrm{nM} \mathrm{CRH}$ and up to $100 \mathrm{nM}$ cortisol (both from Sigma-Aldrich Inc.) for $1 \mathrm{~min}$ to $1 \mathrm{~h}$. The supernatant was carefully removed from the cell layer and centrifuged $\left(800 \times \mathrm{g}, 37^{\circ} \mathrm{C}, 10 \mathrm{~min}\right)$.

\section{FCS}

FCS measurements were performed on a Confocor spectrofluorimeter (Carl Zeiss-Evotec, Jena, Germany) equipped with an air-cooled $488 \mathrm{~nm}$ Argon-laser (LASOS Lasertechnik GmbH, Jena, Germany) and a water immersion objective (C-Apochromat $63 \times / 1.2 \mathrm{~W}$ Korr). The intensity of the laser was set to $70 \mu \mathrm{W}$. Intensity fluctuations were recorded by an avalanche photodiode (SPCM-CD 3017) in photon counting mode, autocorrelated with a hardware correlator (ALV 5000, ALV, Langen, Germany), and analyzed with the FCS ACCESS (Carl Zeiss-Evotec) software package using a multicomponent fit model (see Figure 2).

\section{Focus control and pinhole adjustment}

A drop of the organic fluorescent dye Rhodamine $6 \mathrm{G}$ (diluted 1:200) was used to automatically position the chambered coverglass (Nalge Nunc International, Japan) in focus of the confocal optics of the spectrofluorimeter by a scanning procedure as well as to automatically adjust the pinhole to its correct position. The focus in $z$-direction was set $150 \mu \mathrm{m}$ over the coverglass to record diffusions of fluorescent particles through the focal element in the drop of sample. The pinhole diameter was set to $35 \mu \mathrm{m}$.

\section{Calibration of confocal volume}

The confocal detection volume was determined by measuring the correlation time of a $10-\mathrm{nM}$ solution of rhodamine $6 \mathrm{G}$ in water with the known diffusion coefficient $D$ of $2.8 \times 10^{-6} \mathrm{~cm}^{2} / \mathrm{s}$, employing the relationship $D=r^{2} /\left(4 \tau_{D}\right)$ and resulting in a confocal volume element of $0.17 \mu \mathrm{m}$ in radian and $0.88 \mu \mathrm{m}$ in axial dimension. In addition, DyLight488-labeled monoclonal antibody dilution series with known concentrations (ranging from 1 


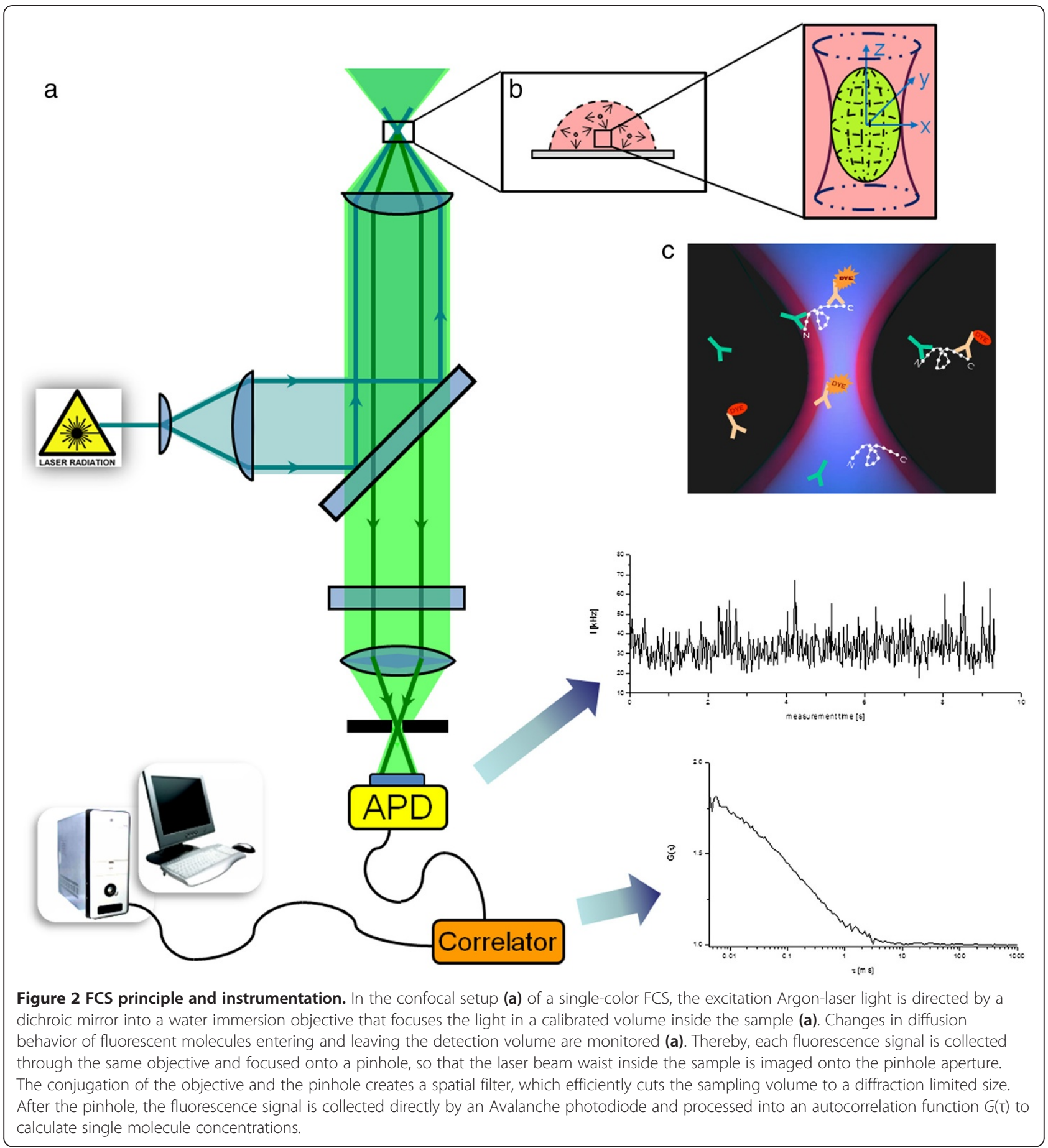

to $80 \mathrm{nM}$ ) were measured and showed similar molarities in FCS. Confocal volume calibrations using rhodamine 6 $G$ were carried out on each experimental day or after $3 \mathrm{~h}$ measurement time.

\section{FCS immunoassay}

$60 \mathrm{nM}$ antiACTH(N-term)-monoclonal IgG1 antibodies (Phoenix Pharmaceuticals Inc., Belmont, USA) and $60 \mathrm{nM-}$ labeled antiACTH(C-term)-IgG1 antibodies (Fitzgerald Industries International, Concord, USA; labeled with DyLight488 from Pierce Biotechnology, Rockford, USA) were added in the cell-free supernatant and incubated $\left(30^{\circ} \mathrm{C}, 15 \mathrm{~min}\right)$ to ensure a quick and absolute ACTHcapture of both monoclonal antibodies. Western blotting analysis and ELISA measurements prior to FCS showed no crossreactivity between both antibodies in absence of 
ACTH. A drop $(25 \mu \mathrm{l})$ of sample was pipetted on the chambered coverglass, excited with an 488-nm Ar-laser attenuated by an optical density filter (1.0-in. diameter), and the fluctuations in fluorescence intensity of the mAb $(N)$-ACTH-mAb $(C)$ immunocomplex compared to the free labeled antibody were monitored in series of 50 measurements with identical setup (measurement time: $10 \mathrm{~s}$; correlator scaling: $10 \mathrm{~s}$ ) for each sample (see Figure 2).

\section{Calculation}

Statistical analysis of the autocorrelation function by a 2-component fitting procedure computationally distinguishes the labeled unbound antibody fraction from the desired ACTH-bound form (immunocomplex). The normalized autocorrelation function $G(\tau)$ describes the fluctuations of a signal $F(t)$ from the mean intensity at any time compared to fluctuations at any later time $F(t+\tau)$. It is given by

$$
G(\tau)=\frac{\langle\delta F(t), \delta F(t+\tau)\rangle}{\langle F(t)\rangle^{2}}
$$

where the angular brackets in the function represent the ensemble average, $\delta F(t)$ denotes the corresponding variance, and $\tau$ is known as the delay or correlation time interval over which the fluctuations are compared.

For a single diffusing species (a one-component model) in a Gaussian confocal volume, the autocorrelation function $G(\tau)$ is defined by [3]

$$
G(\tau)=1+\frac{1}{N\left(1+\frac{\tau}{\tau_{D}}\right) \sqrt{1+\frac{\tau}{\left(\frac{z}{r}\right)^{2} \tau_{D}}}},
$$

where $N$ is the particle number and $\tau_{D}$ the molecular diffusion time of the excited fluorophores moving in a three-dimensional confocal volume through an axial $(z)$ to radial $(r)$ dimension.

The molecular diffusion time for a one photon excitation is given by the following relationship to the diffusion coefficient $D\left[\mathrm{~cm}^{2} / \mathrm{s}\right]$

$$
\tau_{D}=\frac{r^{2}}{4 D}
$$

The obtained autocorrelation functions were evaluated using a two-component model by fixing the diffusion time of the unbound labeled antibody fraction $\left(\tau_{D 1}\right)$ which was achieved from one-component fitting procedure.
The analytical formula for the two-component model, which was successfully applied in a previous work [3], was used in a modified form and is given by

$$
\begin{aligned}
& G(\tau)=1+\frac{1}{N^{\prime}}\left[(1-Y) g_{D 1}(\tau)+Y g_{D 2}(\tau)\right] \\
& \text { with } N^{\prime}=N_{1}+N_{2} \\
& g(\tau)=\left(1+\frac{\tau}{\tau_{D}}\right)^{-1}\left(1+\frac{\tau}{\left(\frac{z}{r}\right)^{2} \tau_{D}^{2}}\right)^{-0.5}
\end{aligned}
$$

This yields values of diffusion times $\left(\tau_{D 1}, \tau_{D 2}\right)$ and of the related mole fractions $Y$ and $(1-Y)$ for the two components. Autocorrelation analysis was performed for a fixed structural parameter of 5 defining the ratio between the height and the width of the detection volume. This parameter was obtained from calibration with rhodamine $6 \mathrm{G}$ in water. The fit model determines the average number of fluorescent molecules within the detection volume, and the characteristic diffusion times. Evaluations of the autocorrelation function of only labeled antibodies result in diffusion times of $\tau_{1}=220 \pm 8 \mu$ s (mean $\pm t$-student) through the confocal volume using a one-component fitting procedure. These results are in accordance to the diffusion time of the IgG-antibody ( $D$ of $3.7 \pm 0.2 \times 10^{-7} \mathrm{~cm}^{2} / \mathrm{s}$ ) of $200 \mu$ s calculated by Stokes-Einstein relation. Formation of the immunocomplex results in a characteristic diffusion behavior of $\tau_{2}=483 \pm 83 \mu$ s (mean $\pm t$ ) through the confocal volume compared to freely labeled anti-ACTH IgG antibody in solution ( $\tau_{1}$ fixed to $\tau_{1}=220 \mu$ s; see Figure 3).

As the amplitude of an autocorrelation function is inversely proportional to the average number of fluorescent particles within the confocal volume ( $V_{\text {conf }}$ of $5 \times$ $\left.10^{-16} \mathrm{l}\right)$

$$
G(0)=\frac{1}{N_{D 1,2}},
$$

the absolute concentration of free labeled IgG antibodies $\left(N_{D 1}\right)$ and of the ACTH immune complexes (equal to number of ACTH molecules; $N_{D 2}=N_{\mathrm{ACTH}}$ ) can be obtained by

$$
\begin{aligned}
c_{\mathrm{ACTH}} & =\frac{N_{\mathrm{ACTH}}}{6.023 \cdot 10^{23} \mathrm{~mol}^{-1} \cdot 5 \times 10^{-16} 1} \\
& =\frac{N_{\mathrm{ACTH}}}{30.115 \times 10^{7}} \mathrm{~mol} / 1 \\
c_{\mathrm{IgG}} & =\frac{N_{\mathrm{IgG}}}{30.115 \times 10^{7}} \mathrm{~mol} / 1
\end{aligned}
$$




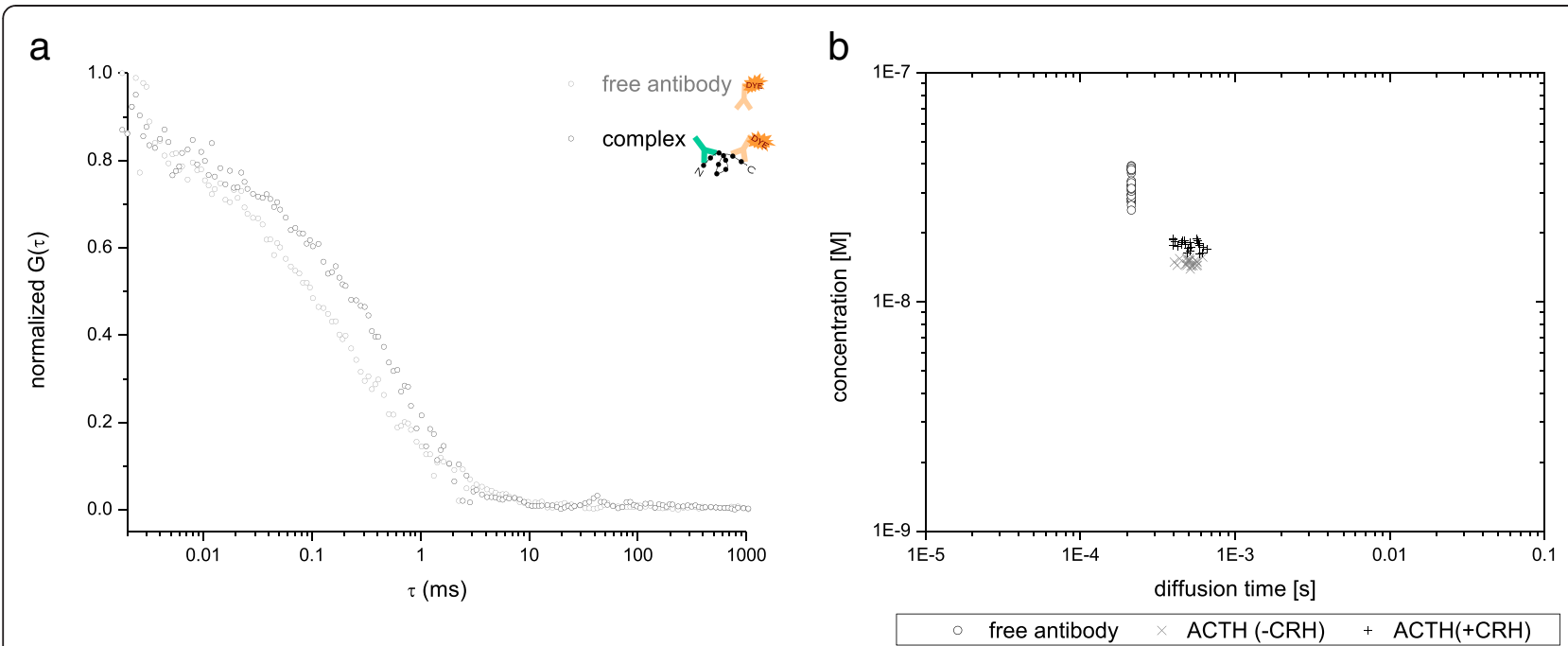

Figure 3 FCS studies of a single and two diffusion species. (a) The two autocorrelation functions were calculated from intensity fluctuations of the free antibody and that of the ACTH-bound ones. The shift of the autocorrelation curve (a, blue line) to the right indicates that the diffusion time of the ACTH-bound particles through the focus is higher than that of the free antibody. To eliminate the amount of unbound from the ACTH-bound forms in the sample, a two-component fit over the autocorrelated data points of the ACTH-bound form (a, blue line) was performed with a fixed diffusion time for the unbound antibodies (220 $\mu \mathrm{s}$, previously calculated with a one-component fit to obtain the average diffusion time of free unbound fluorescent antibodies) and a variable one for quantifying different ACTH concentrations. (b) A double-logarithmic plot of calculated concentrations versus related diffusion times over 50 measurements shows the accuracy of the results obtained by FCS for different ACTH concentrations.

\section{Results}

\section{Dose response relation of CRH and cortisol to ACTH secretion}

The experimental results show a basal ACTH secretion which is not affected by extracellular CRH and cortisol signals (see Figure 4) and thus it seems not to be modulated by the main feedback controls. Moreover, our data feature a strong ACTH response $(17.427 \pm 0.422$ $\mathrm{nM})$ to an extracellular dose of $10 \mathrm{nM}$ CRH over $22 \mathrm{~h}$ (compared to that of 5 and $36 \mathrm{nM}$ ), indicating an increased ACTH secretion in the AtT-20 cells in vitro. In addition to an extracellular dose of 10-nM CRH, an
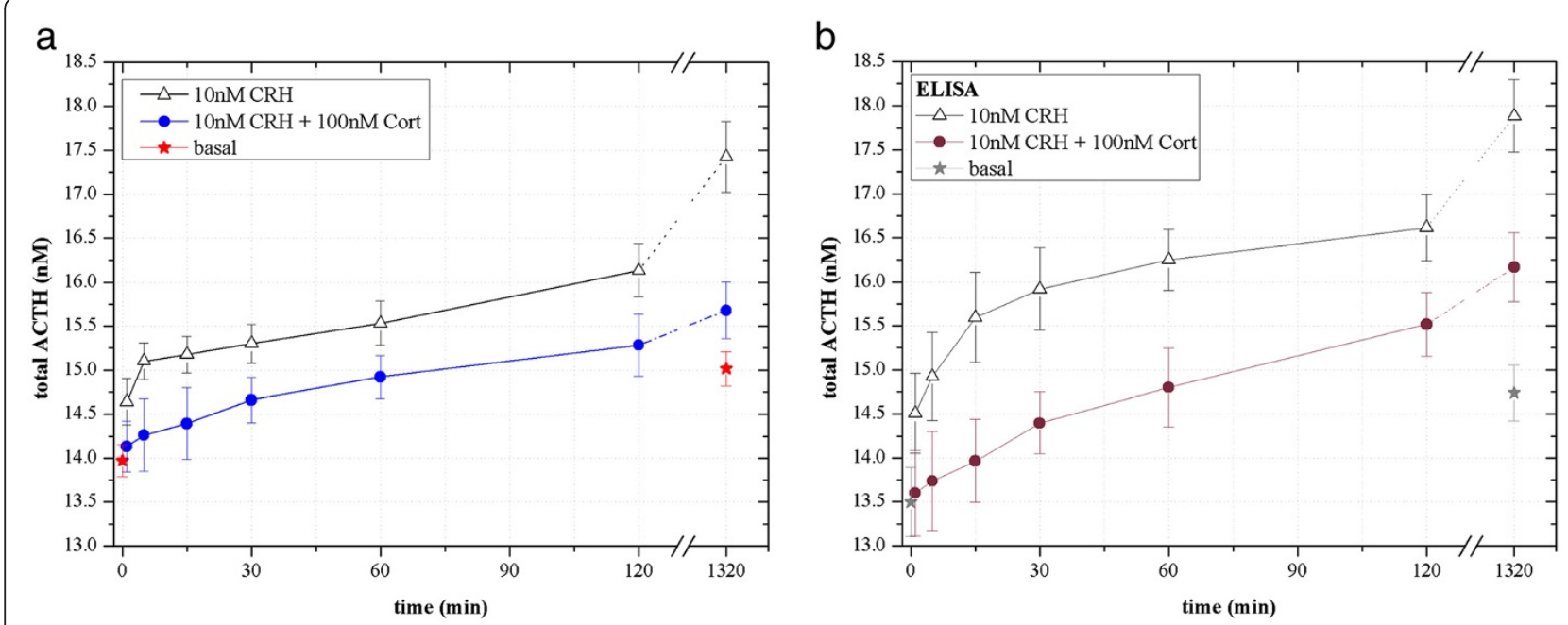

Figure 4 Fast and delayed negative feedback regulation of ACTH release in vitro. A typical HPA response to a strong extracellular stress signal (10-nM CRH) is simulated in AtT-20 pituitaries in vitro by adding 100-nM cortisol. Already minutes after cortisol incubation an inhibition and a delay of extracellular ACTH secretion is detectable (blue circular data points), which is supposed to be caused by a fast negative feedback mechanism. Long-term inhibitory effects of cortisol to ACTH secretion (interrupted line) were not analyzed. FCS datasets (a) are consistent with ELISA control measurements (b). Deviations calculated by the $t$-test distribution, $a=0.1$. 
administration of $50 \mathrm{nM}$ cortisol partly inhibited the stimulated ACTH secretion after $22 \mathrm{~h}$ compared to that in absence of cortisol. A maximal inhibition of the ACTH release was achieved by adding 100-nM cortisol leading to ACTH levels close to the basal one (Table 1).

\section{Fast feedback regulation of ACTH release}

In order to demonstrate the capabilities of the method we focused on the fast negative feedback control by cortisol. Due to the fast sampling and the low sample volume we were able to detect significant differences in ACTH response within 5-15 min after CRH and/or cortisol incubation (see Figure 4).

\section{Validation of FCS results}

FCS and immunoassays (with chemiluminescent, fluorescent, or HRPO signals) are both known as quite sensitive detection techniques. Thus, a two-site ELISA (MDBioscience, Switzerland) is used to validate the data obtained by FCS. Table 2 and Figure 4 show that the ELISA results are consistent with the detected FCS datasets.

\section{Mathematical modeling of feedback mechanisms}

The improved setup for the FCS method is particularly suitable for experiments which have to be conducted repeatedly and demand a high (quantitative) accuracy of the data. A central motivation to develop such a technique comes from a mathematical modeling task initiated by the research presented in [9]. We are interested in the interplay of the genomic and non-genomic negative feedback of cortisol on the secretion of ACTH and its effect on the dynamics of the HPA-axis. This research goal demands to model both intracellular mechanisms as well as interactions of the different glands. This cannot be achieved in full detail. Consequently, we concentrated on the main feedback mechanisms related to the anterior pituitary gland and the basic controls between the hypothalamus and the adrenal

Table 1 In vitro studies in AtT-20s within 22 h

\begin{tabular}{llll}
\hline CRH $(\mathrm{nM})$ & Cort $(\mathrm{nM})$ & Cell population (cells/ml) & ACTH $(\mathrm{nM})$ \\
\hline 0 & 0 & $205164 \pm 12889$ & $15.016 \pm 0.201$ \\
5 & 0 & $335938 \pm 20522$ & $16.764 \pm 0.593$ \\
$\mathbf{1 0}$ & $\mathbf{0}$ & $324219 \pm 23935$ & $\mathbf{1 7 . 4 2 7} \pm \mathbf{0 . 4 2 2}$ \\
36 & 0 & $302084 \pm 28168$ & $16.146 \pm 0.564$ \\
54 & 0 & $237500 \pm 15630$ & $15.364 \pm 0.575$ \\
10 & 50 & $312500 \pm 28125$ & $16.673 \pm 0.467$ \\
$\mathbf{1 0}$ & $\mathbf{1 0 0}$ & $298438 \pm 20625$ & $\mathbf{1 5 . 6 7 9} \pm \mathbf{0 . 3 3 7}$ \\
\hline
\end{tabular}

Cell proliferation and ACTH response to various doses of extracellular CRH and cortisol within/over $22 \mathrm{~h}$ of incubation ( $114 \mathrm{~h}$ total incubation time). Optimal concentrations for CRH stimulation and cortisol inhibition of ACTH secretion were used for studying feedback regulation of ACTH release (bold values). Deviations calculated by the $t$-test distribution, $\mathrm{a}=0.1$.
Table 2 Validation of FCS results

\begin{tabular}{|c|c|c|c|c|}
\hline \multirow{2}{*}{$\begin{array}{l}\text { Time } \\
\text { (min) }\end{array}$} & \multicolumn{2}{|l|}{ ACTH (FCS) } & \multicolumn{2}{|l|}{ ACTH (ELISA) } \\
\hline & $\%$ change $^{a}$ & $\%$ dev & $\%$ change to FCS & $\% \mathrm{dev}$ \\
\hline \multicolumn{5}{|l|}{ Basal } \\
\hline 0 & 0.00 & \pm 1.28 & -3.39 & \pm 2.91 \\
\hline 1320 & +7.48 & \pm 1.29 & -1.86 & \pm 2.16 \\
\hline \multicolumn{5}{|c|}{$+10 \mathrm{nM} \mathrm{CRH}$} \\
\hline 1 & +4.79 & \pm 1.82 & -0.91 & \pm 3.11 \\
\hline 5 & +8.08 & \pm 1.39 & -1.16 & \pm 3.35 \\
\hline 15 & +8.62 & \pm 1.36 & +2.77 & \pm 3.29 \\
\hline 30 & +9.51 & \pm 1.43 & +4.05 & \pm 2.96 \\
\hline 60 & +11.18 & \pm 1.62 & +4.61 & \pm 2.12 \\
\hline 120 & +15.50 & \pm 1.86 & +2.97 & \pm 2.27 \\
\hline 1320 & +24.74 & \pm 2.32 & +2.63 & \pm 2.31 \\
\hline \multicolumn{5}{|c|}{ +10 nM CRH + 100 nM cortisol } \\
\hline 1 & +1.16 & \pm 2.03 & -3.77 & \pm 3.58 \\
\hline 5 & +2.08 & \pm 2.88 & -3.68 & \pm 4.12 \\
\hline 15 & +3.02 & \pm 2.85 & -2.97 & \pm 3.38 \\
\hline 30 & +4.94 & \pm 1.75 & -1.80 & \pm 2.44 \\
\hline 60 & +6.80 & \pm 1.63 & -0.81 & \pm 3.01 \\
\hline 120 & +9.40 & \pm 2.31 & +1.52 & \pm 2.33 \\
\hline 1320 & +12.23 & \pm 2.07 & +3.10 & \pm 2.41 \\
\hline
\end{tabular}

Validation of FCS results by ELISA measurements. The \% difference in ACTH concentrations obtained from ELISA measurements refers to the FCS dataset of the same sample (in the same row in the table).

${ }^{\text {a }}$ The temporal \% change of ACTH concentration to the basal ACTH-level of $13.9709 \mathrm{nM}$ (mean value) detected by FCS. Deviations calculated by the $t$-test distribution, $a=0.1$.

glands. This approach of bridging several levels of complexity eventually needs validation by experimental data. In that regard the measurement of the $\mathrm{ACTH}$ secretion in response to $\mathrm{CRH}$ and cortisol serves two goals. First of all the secretion of ACTH is targeted by the main feedback controls we focus on in our model and thus allows us at least a basic assessment of the model. And secondly, we introduce no bias concerning the overall dynamics of the HPA-axis, as we consider only the anterior pituitary cells in our in vitro experiment and thus have no interaction with other tissues or glands.

With respect to the modeling technique we followed an approach in [10] which also focused on the anterior pituitary cells. Figure 5 provides a sketch of the considered feedback controls and the used mathematical equations in [10]. In Figure 6, we provide a graphical outline of our model and the corresponding mathematical description as set of the ordinary differential equations. Our extended model follows the approach offered in [10] but in addition considers the fast non-genomic feedback mechanism via the glucocorticoid membrane receptor in the anterior pituitary cells (red-framed pathway). Moreover, the model includes the slow genomic 


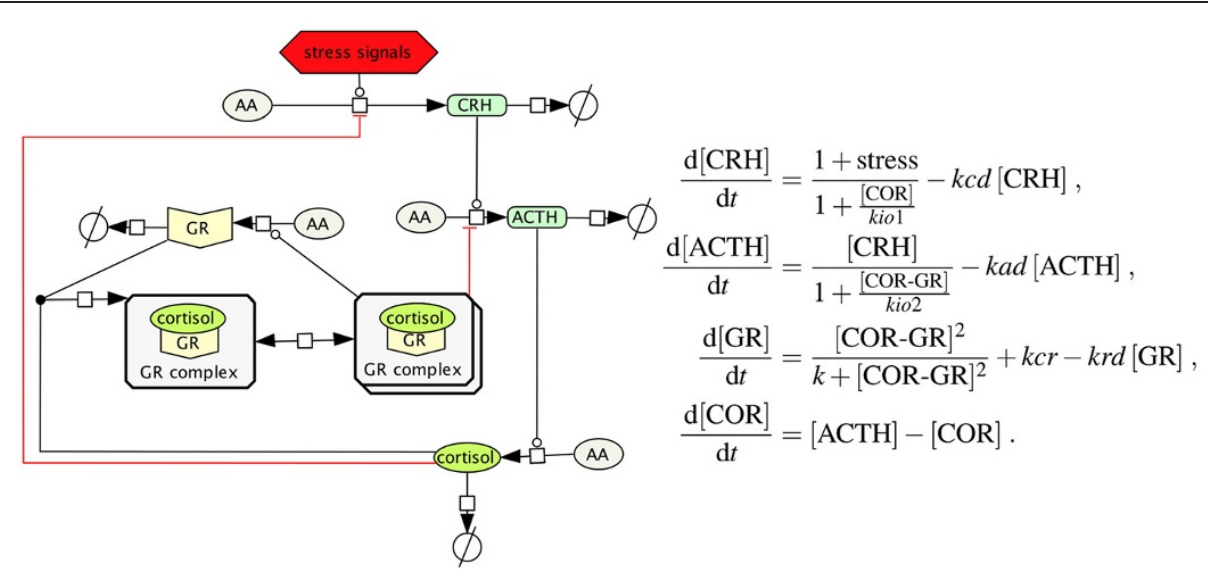

Figure 5 Parsimonious model of the HPA-axis. The figure shows a graphical outline and the corresponding mathematical equations of the parsimonious model as published in [10]. The focus of this model is the negative feedback of cortisol on the production of ACTH via an intracellular glucocorticoid receptor, which acts as a transcription factor in its dimerized form. Eventually, the model offers an intriguing explanation for the widely observed scenario of hypocortisolism.

feedback mechanism of cortisol (green-framed pathway) and $\mathrm{CRH}$-mediated genomic and non-genomic effects (blue-framed pathway). To the best of the authors' knowledge the proposed model is the first one of the HPA-axis which incorporates central receptors. Consequently, it differs significantly in size and structure from most models present in literature, which focus on most prominent species cortisol, $\mathrm{ACTH}, \mathrm{CRH}$, and vasopressin (cf. [11-14]). Moreover, our model is novel in the sense that we explicitly model an intracellular compartment and the nucleus. This allows us to take translocation processes into account and thus differ between genomic and non-genomic effects.

In a first attempt we used the model from [10] and tried to reproduce the data obtained by means of our FCS method. Figure 7 shows that if we consider only one of the conducted experiments, i.e., only a dosage of $\mathrm{CRH}$ or the scenario of adding CRH as well as cortisol, the model is

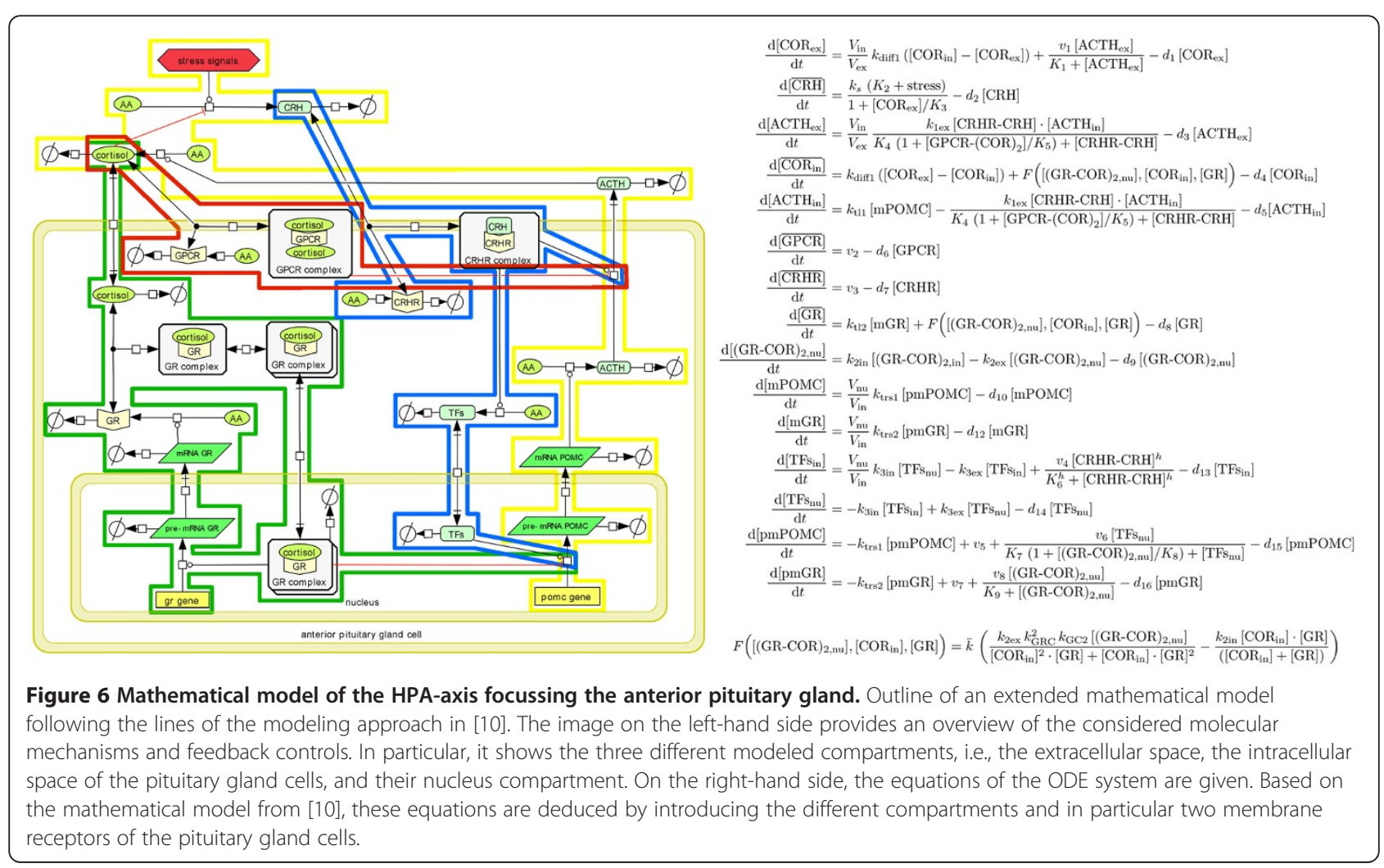



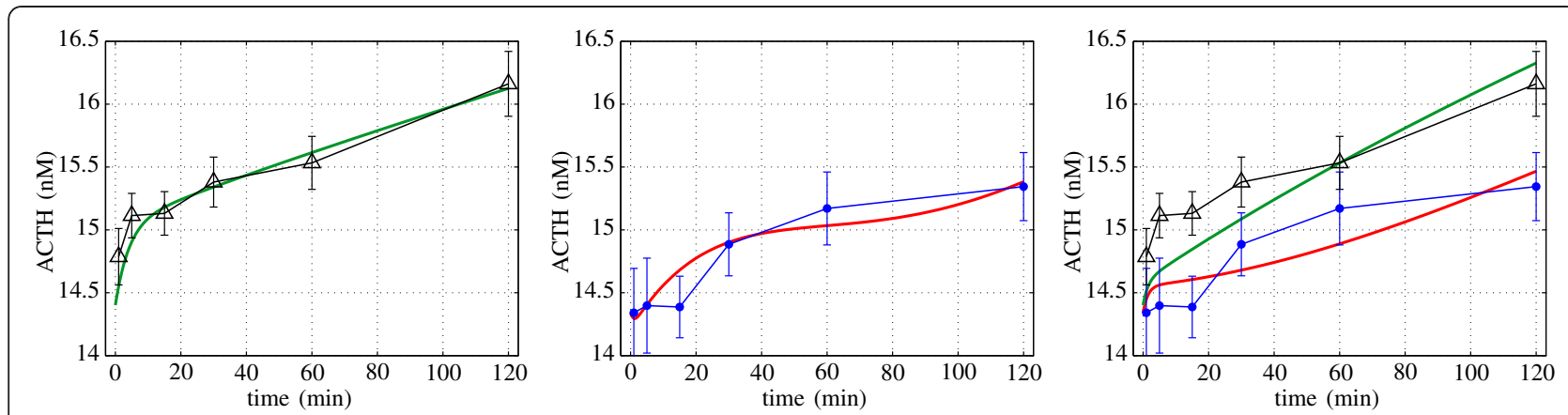

$\longrightarrow$ reconstruction data $\mathrm{A} \longrightarrow$ data $\mathrm{A}(+10 \mathrm{nM}$ CRH, +100nM cortisol)

reconstruction data $\mathrm{B}$

$\triangle$ data B $(+10 \mathrm{nM} \mathrm{CRH})$

Figure 7 Fit of the experimental data by means of the parsimonious model from [10]. The figure shows the result of a numerical experiment for the obtained experimental data and the mathematical model as published in [10]. The first (left) plot shows the result of a parameter fit for the dataset without added cortisol. The second (middle) plot shows the reproduction of the ACTH response curve in the presence of $\mathrm{CRH}$ and cortisol. The last (right) plot presents the result when considering both experimental datasets at once. The poor quality of the obtained fit in the latter case indicates that the parsimonious model is not capable to reproduce the fast and slow dynamics related to the negative feedback via cortisol.

capable to reproduce the basic dynamics. However, we were not able to find any parameter set such that the parsimonious model is able to exhibit both scenarios. The parameter space was searched using the genetic algorithms from the MATLAB global optimization toolbox. In particular, we used different initial populations of about $10^{4}$ to $10^{5}$ elements. The objective functional was based on Tikhonovtype regularization functional (c.f. [9] for instance).
Figure 8 shows the data fit computed for our extended model. The parameter fit was computed by the Tikhonov regularization, where we used standard global and local optimization algorithms to minimize the objective functional. The extended model allows to correctly reproduce the observed ACTH response behavior. The fact that the model is capable to reproduce the experimental data indicates at least a feasible model and, particularly, supports

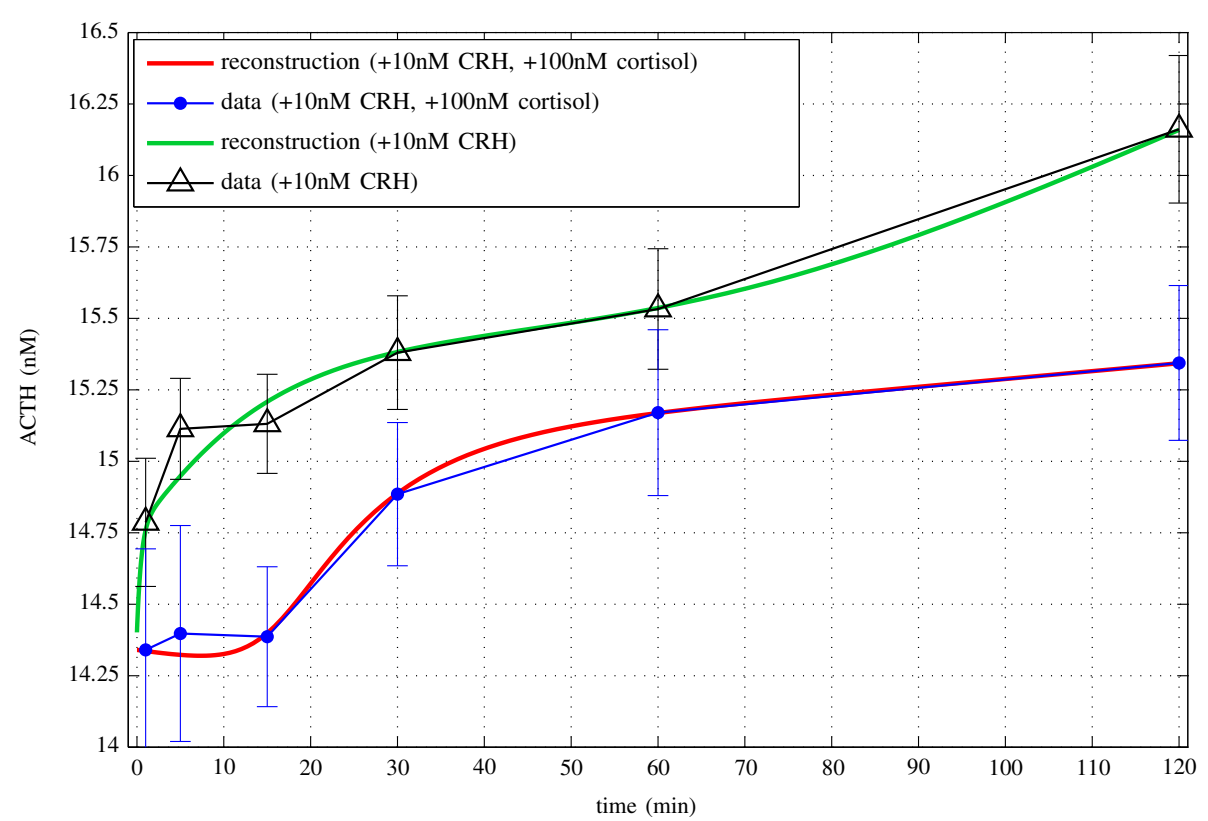

Figure 8 Fit of the experimental data by means of the multicompartment model. The graph shows the result of the parameter fitting for the extended mathematical model, including the membrane receptors for cortisol and CRH. The identified parameters allow to reproduce the observed dynamics in the two scenarios, i.e., with and without cortisol. Hence, by using different initial values for the ODE state variable both characteristic dynamics can be simulated with the same set of parameters. In particular, in the presence of cortisol the fast negative feedback can be reproduced, leading to the experimentally observed sigmoidal curve. 
Table 3 FCS-immunoassay versus ELISA

\begin{tabular}{|c|c|c|}
\hline Parameter & FCS-assay & ELISA \\
\hline Sensitivity & $\mu \mathrm{M}$ to sub-nM & $\mathrm{nM}$ to sub-nM \\
\hline $\begin{array}{l}\text { Sample volume } \\
\text { required }\end{array}$ & Approximately $20 \mu \mathrm{l}$ & $100 \mu l$ \\
\hline Measurement time & Approximately 25 min & $>2.5 h$ \\
\hline $\begin{array}{l}\text { Immunocomplex } \\
\text { formation }\end{array}$ & In solution & On the surface \\
\hline $\begin{array}{l}\text { Washing steps } \\
\text { required }\end{array}$ & No & Yes \\
\hline $\begin{array}{l}\text { Calibration curve } \\
\text { required }\end{array}$ & Yes (confocal volume) & Yes \\
\hline Detection mode & $\begin{array}{l}\text { Size-based fluctuations in } \\
\text { signal intensities }\end{array}$ & $\begin{array}{l}\text { Changes in signal } \\
\text { intensity }\end{array}$ \\
\hline
\end{tabular}

the underlying idea of distinguishing between the genomic and non-genomic feedback mechanisms. We emphasize that the present dataset is neither sufficient nor suited to identify the 'true' values of all model parameters. The conducted numerical experiment mainly serves to assess the overall behavior of the extended model, particularly in comparison with the parsimonious model as discussed in [10].

\section{Conclusion}

In 2005, Maier et al. [3] were able to provide evidence of a glucocorticoid receptor in the anterior pituitary cell membrane which may regulate fast response of anterior pituitary cells to cortisol. With our improved FCS setup it was possible to detect lowest changes in extracellular ACTH molarities $( \pm 0.3 \mathrm{nM})$ that arise from signaling of these activated G-protein coupled membrane receptors in vitro (see Figure 1). Even 5-15 min after cortisol administration $(100 \mathrm{nM})$ we were able to monitor an inhibition of CRH-induced ACTH secretion by cortisol. Extracellular ACTH levels of $14.387 \pm 0.428 \mathrm{nM}$ compared to $15.131 \pm 0.254 \mathrm{nM}$ without addition of cortisol were measured. The fast inhibitory effects on CRHinduced ACTH secretion have become evident within at most 5 min after cortisol administration (see Figure 4). However, a detailed temporal restriction of fast and slower feedback actions on extracellular ACTH secretion was not studied with this FCS setup so far, but our results suggested that immediate ACTH secretion which has occurred within minutes after cortisol treatment can only be caused by fast non-genomic feedback actions (see Figure 1) and not by genomic-slow feedback mechanisms which have been shown to occur after several hours [15].

FCS provides a highly flexible, easy-to-use assay format with very small sample volumes (approximately 20 $\mu \mathrm{l})$, and increased throughput, as particle numbers can be measured directly after calibrating the confocal volume once, a major disadvantage of ELISAs which need to be calibrated quite often. In addition, FCS makes it possible to extract complex signals from high background due to the different characteristic time scales over which signal and noise occur.

In conclusion, we have demonstrated that this improved FCS setup can be used for fast and sensitive detection of a specific peptide hormone in vitro. By means of the mentioned model system we established this solution-based single molecule detection technique as an alternative to the commonly used approaches, such as ELISAs (with fluorescent, chemiluminescent, or HRPO signal), with respect to rapidity and sensitivity (Table 3). It was possible to even detect nanomolar changes in ACTH secretion with deviations of only 0.2-0.7 $\mathrm{nM}$ approximately in response to an extracellular stress signal over a short period of time. The quality of the data obtained by FCS allowed to study fast feedback mechanisms in the HPA-axis regulatory system in vitro and allowed to support the development of a mathematical model of that HPA-axis network. As opposed to [10] our model takes both the genomic and non-genomic feedbacks mechanisms into account. As a result it is able to feature both ACTH response curves with a single set of model parameters.

\section{Abbreviations}

ACTH: Adrenocorticotropic hormone; $\mathrm{CRH}$ : Corticotrophin-releasing hormone; CRHR: CRH-receptor; ELISA: Enzyme-linked immunosorbent assay;

FCS: Fluorescence correlation spectroscopy; GPCR: G-protein-coupled receptor; GR: Glucocorticoid receptor; HPA: Hypothalamic-pituitary-adrenal; mAb: Monoclonal antibody; POMC: Pro-opiomelanocortin; TF: Transcription factor.

Competing interests

The authors declare that they have no competing interests.

\section{Acknowledgments}

This study was supported by the Vienna Science and Technology Fund (WWTF) Grant no. MA07-030.

\section{Author details}

${ }^{1}$ Department of Structural and Computational Biology, Max F. Perutz Laboratories (MFPL), University of Vienna, Campus-Vienna-Biocenter 5, Vienna 1030, Austria. ${ }^{2} J o h a n n$ Radon Institute for Computational and Applied Mathematics (RICAM), Austrian Academy of Sciences, Altenbergerstr. 69, Linz 4040, Austria.

Received: 21 December 2011 Accepted: 2 October 2012 Published: 26 October 2012

\section{References}

1. JC Buckingham, HC Christian, GE Gillies, JG Philip, AD Taylor, in The Physiology of Immunity, ed. by M.D. Kendall, J.A. Marsh (CRC Press, USA, 1996), p. 331

2. B Hinz, R Hirschelmann, Rapid non-genomic feedback effects of glucocorticoids and CRF-induced ACTH secretion in rats. Pharm. Res. 17, 1273-1277 (2000). doi:10.1023/A:1026499604848

3. C Maier, D Rünzler, J Schindelar, G Grabner, W Waldhäusl, G Köhler, A Luger, G-protein-coupled glucocorticoid receptors on the pituitary cell membrane. J. Cell. Sci. 118, 3353-3361 (2005). doi:10.1242/jcs.02462

4. J Wruss, D Rünzler, C Steiger, P Chiba, G Köhler, D Blaas, Attachment of VLDL receptors to an icosahedral virus along the 5 -fold symmetry axis: multiple binding modes evidenced by fluorescence correlation 
spectroscopy. Biochemistry 46(21), 6331-6339 (2007). doi:10.1021/ bi700262w

5. JC Politz, ES Browne, DE Wolf, T Pederson, Intranuclear diffusion and hybridization state of oligonucleotides measured by fluorescence correlation spectroscopy in living cells. Proc. Natl Acad. Sci. USA 95, 60436048 (1998). doi:10.1073/pnas.95.11.6043

6. H Glauner, IR Ruttekolk, K Hansen, B Steemers, YD Chung, F Becker, S Hannus, R Brock, Simultaneous detection of intracellular target and offtarget binding of small molecule cancer drugs at nanomolar concentrations. Br. J. Pharmacol. 160, 958-970 (2010). doi:10.1111/j.14765381.2010.00732.x

7. L Tang, C Dong, J Ren, Highly sensitive homogenous immunoassay of cancer biomarker using silver nanoparticles enhanced fluorescence correlation spectroscopy. Talanta 81, 1560-1567 (2010). doi:10.1016/j. talanta.2010.03.002

8. K Bacia, P Schwille, Fluorescence correlation spectroscopy. Methods Mol. Biol. 398, 73-84 (2007). doi:10.1007/978-1-59745-513-8_7

9. HW Engl, C Flamm, P Kügler, J Lu, S Müller, P Schuster, Inverse problems in systems biology. Inverse Problems 25, 123014 (2009). doi:10.1088/0266$5611 / 25 / 12 / 123014$

10. S Gupta, E Aslakson, BM Gurbaxani, SD Vernon, Inclusion of the glucocorticoid receptor in a hypothalamic pituitary adrenal axis model reveals bistability. Theor. Biol. Med. Model 4(8) (2007). doi:10.1186/17424682-4-8

11. V Kyrylov, LA Severyanova, A Vieira, Modeling robust oscillatory behavior of the hypothalamic-pituitary-adrenal axis. IEEE Trans. Biomed. Eng. 52(12), 1977-1983 (2005)

12. G Li, B Liu, Y Liu, A dynamical model of the pulsatile secretion of the hypothalamo-pituitary-thyroid axis. Biosystems 35(1), 83-92 (1995)

13. BZ Liu, JH Peng, YC Sun, YW Liu, A comprehensive dynamical model of pulsatile secretion of the hypothalamo-pituitary-gonadal axis in man. Comput. Biol. Med. 27(6), 507-513 (1997)

14. D Savic, S Jelic, A mathematical model of the hypothalamo-pituitaryadrenocortical system and its stability analysis. Chaos Solitons Fract. 26, 427-436 (2005)

15. O Wang, JA Majzoub, in The Pituitary, ed. by M. Shlomo (Elsevier, Amsterdam, 2011), p. 68

doi:10.1186/1687-4153-2012-17

Cite this article as: Puchinger et al:: In vitro detection of adrenocorticotropic hormone levels by fluorescence correlation spectroscopy immunoassay for mathematical modeling of glucocorticoid-mediated feedback mechanisms. EURASIP Journal on Bioinformatics and Systems Biology 2012 2012:17.

\section{Submit your manuscript to a SpringerOpen ${ }^{\circ}$ journal and benefit from:}

- Convenient online submission

- Rigorous peer review

- Immediate publication on acceptance

- Open access: articles freely available online

- High visibility within the field

- Retaining the copyright to your article

Submit your next manuscript at $\gg$ springeropen.com 